\title{
Surgical repair of concomitant ventricular septal defect and aortic cusp prolapse or aortic regurgitation, also known as the Laubry-Pezzi syndrome
}

\author{
Margaux Pontailler, Régis Gaudin, Anne Moreau de Bellaing, Olivier Raisky \\ Department of Pediatric Cardiac Surgery, Necker Sick Children Hospital-M3C and University Paris Descartes, Paris, France \\ Correspondence to: Olivier Raisky, MD, PhD. Department of Pediatric Cardiac Surgery, Necker Sick Children Hospital and Paris Descartes University, \\ 149 rue de Sèvres, 75015 Paris, France. Email: olivier.raisky@aphp.fr.
}

Submitted May 06, 2019. Accepted for publication: May 09, 2019.

doi: 10.21037/acs.2019.05.11

View this article at: http://dx.doi.org/10.21037/acs.2019.05.11

\section{Introduction}

It has been almost a century since Charles Laubry and Cesare Pezzi first described the clinical features of aortic regurgitation (AR) due to aortic valve prolapse in patients with ventricular septal defects (VSDs) (1). The Venturi effect is the explanation to Laubry-Pezzi syndrome: a significant fall in pressure when blood flows through the VSD under the aortic valve, sucking either the right or the non-coronary leaflet in the VSD, resulting in AR. It is most commonly found in infundibular VSDs but can also be encountered with perimembranous VSDs.

\section{Clinical vignette}

A 3 years old male patient, part of a humanitarian program, was initially addressed to our department of pediatric cardiac surgery for surgical closure of a perimembranous VSD. The patient had no past medical history and VSD had been diagnosed postnatally. A diastolic murmur could be heard at physical examination. Preoperative transthoracic echocardiography (TEE) revealed grade 3 (AR) and severe left ventricle (LV) dilatation with preserved $\mathrm{LV}$ function ( $\mathrm{LV}$ ejection fraction 62\%). AR was mainly caused by prolapse of the non-coronary cusp in the VSD.

\section{Surgical technique}

\section{Preparation}

The patient is positioned supine and a transesophageal echocardiography probe is inserted before surgery is initiated. Surgical repair is performed under general anaesthesia via a median sternotomy, with standard aortic and bicaval venous cannulation and normothermic cardiopulmonary bypass. LV venting is inserted in the upper right pulmonary vein.

\section{Exposition}

Transverse aortotomy allows direct administration of the blood cardioplegia in the coronary ostia (repeated every 10 minutes) and analysis of the regurgitant aortic valve. Three 5/0 stitches are placed on each commissure to allow better visualisation of the aortic valve. Each cusp of the aortic valve undergo geometric and effective height assessments, alongside assessment of the commissures.

\section{Operation}

The nadir of the non-coronary aortic leaflet is carefully delaminated from its abnormal attachment to the VSD. Once freed, the leaflet is partially detached from the annulus: we start from the right/non-coronary commissure and leave the leaflet only attached by the left/noncoronary commissure. This allows visualizing the VSD via the aortotomy. The VSD is then directly closed using separated mattresses sutures running from the ventricular septal crest to the aortic root. Once the VSD is closed, the non-coronary cusp is reinserted: first of all, the remaining commissure is resuspended, then the opposite commissure is reinserted after examination of the length of the free margin; the aortic margin is then reinserted just above the 
neo-annulus (fibrous zone created by the direct closure of the VSD) using two running sutures, from the commissures to the nadir of the cusp. This technique can avoid the distortion of the nadir of the cusp, which is seen when direct closure of the VSD is performed without detachment of the cusp. Adjacent cusps' free edges are then aligned using stitches of Prolene 6/0 to examine the residual prolapse of the non-coronary cusp: leaflet plication with central stitches in order to shorten the free margin of the prolapsing aortic valve can be performed if deemed necessary and final examination consisting of the measurement of the effective height with a dedicated caliper is carried out (Schäfers caliper).

\section{Completion}

The surgery was uneventful. The patient was weaned from mechanical ventilation 4 hours after surgery, inotropic support was stopped after 24 hours, and the patient was discharged from hospital 5 days after surgery. At discharge, aortic regurgitation was found to be either nil or trivial and LV dilatation had significantly decreased at echocardiographic examination.

\section{Comments}

\section{Clinical results}

It is commonly accepted that early closure of the VSD should be performed before onset of AR to prevent its occurrence and to prevent progression of $\mathrm{AR}$ when present before surgical repair. Most authors describe concomitant VSD closure and aortic valvuloplasty by shortening of the free margin of the prolapsing aortic valve when VSD is associated with cusp prolapse or AR. Historical cohorts report a rate of aortic valvuloplasty failure up to $25 \%$ at 10 years after surgical correction of VSD with concomitant $\operatorname{AR}(2,3)$.

\section{Advantages and caveats}

Laubry-Pezzi syndrome does not only implicate leaflet prolapse, but also aortic cusp retraction, aortic annulus dilatation and deformation of the adjacent sinus of Valsalva. Damages to the aortic valve are irreversible and AR will keep on worsening without surgery. Progression to severe AR leads to aortic valve replacement, or even Ross or Bentall procedures when the sinuses are abnormally deformed. Brizard et al. associated direct closure of the VSD after mobilization of the cusp and insertion of a patch in the sinus of Valsalva in half of their patients to avoid later deformation (4). Zhao et al. described the use of an "8-shaped patch" for correction of aortic valve prolapse and VSD closure in Laubry-Pezzi syndrome: the aim of the upper half of the patch is to reinforce, support and elevate the Valsalva sinus, while the lower half is used to close the VSD (5). The prolapsing leaflet is then divided and sutured using an overlapping technique.

The technique we describe here allows concomitant VSD closure, creation of a fibrous zone just beneath the pathologic cusp, realignment of the septal crest and aortic valvuloplasty via a single transaortic approach. Meticulous analysis of the prolapsing mechanism combined with careful mobilization-to increase the aortic leaflet geometric height-and resuspension of the responsible leafletto increase the aortic leaflet effective height-are the key determinants in treating patients with severe AR and aortic cusp distortion. Our strategy aims to combine the advantage of VSD direct closure, offering a significant fibrous support for the aortic valve and a perfect realignment of the septal crest without the adverse down attraction of the nadir of the cusp towards the LV. Nevertheless, this aggressive technique only concerns in our experience severely deteriorated aortic valves. In the case of mild AR, moderate prolapse and a normal geometric height, we are still proposing a traditional surgery with resuspension at the level of the commissure and/or at the level of the nodules of Arantius.

Whatever the initial repair consisted of, patients treated for VSD and AR require a close long-term follow-up of both the aortic valve and the aortic root, especially when preoperative $\mathrm{AR}$ was moderate to severe and when surgery occurred at an older age.

\section{Acknowledgments}

None.

\section{Footnote}

Conflicts of Interest: The authors have no conflicts of interest to declare.

\section{References}

1. Laubry C, Pezzi C. Traité des maladies congénitales du 
coeur. Paris: JB Bailliere; 1921. Quoted by Labury C, Routier D, Soulie P. Les souffles de la maladie de Roger. Rev Med Paris 1933;50:439-48.

2. Rhodes LA, Keane JF, Keane JP, et al. Long follow-up (to 43 years) of ventricular septal defect with audible aortic regurgitation. Am J Cardiol 1990;66:340-5.

3. Trusler GA, Williams WG, Smallhorn JF, et al. Late results after repair of aortic insufficiency associated with ventricular septal defect. J Thorac Cardiovasc Surg

Cite this article as: Pontailler M, Gaudin R, Moreau de Bellaing A, Raisky O. Surgical repair of concomitant ventricular septal defect and aortic cusp prolapse or aortic regurgitation, also known as the Laubry-Pezzi syndrome. Ann Cardiothorac Surg 2019;8(3):438-440. doi: 10.21037/acs.2019.05.11
1992;103:276-81.

4. Brizard C. Surgical repair of infundibular ventricular septal defect and aortic regurgitation. Semin Thorac Cardiovasc Surg Pediatr Card Surg Annu 2006:153-60.

5. Zhao T, Hu J, Yang Y. Anatomic and functional aortic valvuloplasty for correction of aortic valve prolapse in ventricular septal defect with aortic insufficiency. Ann Thorac Surg 2011;91:308-10. 